\title{
Cardiac emergencies in neonates and young infants
}

\author{
Nasib Kabbani, Mohamed S. Kabbani ${ }^{1}$, Hayan Al Taweel ${ }^{1}$
}

Alfaisal University, Riyadh, Kingdom of Saudi Arabia, ${ }^{1}$ Cardiac Sciences, Pediatric Cardiac ICU Section, King Abdulaziz Medical City, Ministry of National Guard Health Affairs, Riyadh, Kingdom of Saudi Arabia

\begin{tabular}{|c|}
\hline Access this article online \\
\hline Website: www.avicennajmed.com \\
\hline DOI: 10.4103/2231-0770.197506 \\
\hline Quick Response Code: \\
\hline
\end{tabular}

\begin{abstract}
Cardiac emergencies in children are not infrequent. Early recognition and management are essential to save life and prevent any comorbidity. The presentation of cardiac emergencies and etiologies is variable depending on the age of child at the time of presentation and type of cardiac lesion. Cyanotic and noncyanotic congenital heart diseases are the main causes in neonates and infants. Acquired heart diseases and dysrhythmia are more common causes for cardiac emergencies in toddler and childhood. In this review, we discuss the most common causes for cardiac emergencies in neonates and young infants highlighting important points in the presentation and management that are essential for early recognition and timely management of infants presenting with these conditions.
\end{abstract}

Key words: Cardiac surgery, congenital heart disease, neonatal cardiac emergencies

\section{INTRODUCTION}

The incidence of congenital heart diseases is reported in $7-9 / 1000$ live birth with $1 / 6^{\text {th }}$ to $1 / 4^{\text {th }}$ of these cases having critical congenital heart diseases. ${ }^{[1]}$ Neonates with cardiac disease may present in acute or critical condition. Although cardiac diseases are not common causes among general pediatric emergencies, ${ }^{[2]}$ it is highly important to recognize these causes early and initiate proper treatments as they need specific and urgent management. Late diagnosis can lead to significant respiratory compromises or circulatory failure in up to $30 \%$ of nonsyndromic children born with congenital heart diseases. ${ }^{[1]}$ The etiology of pediatric cardiac emergencies is variable based on the age at presentation and the cause leading to emergency whether congenital or acquired heart diseases.

In this line, we can identify congenital heart lesions as main reasons for a cardiac emergency in neonates and young infants while acquired heart diseases become more common later on in infancy and early childhood.

Furthermore, congenital cardiac diseases leading to emergencies can be classified into cyanotic and noncyanotic

Address for correspondence: Dr. Mohamed S. Kabbani, King Abdulaziz Medical City, Ministry of National Guard Health Affairs, Riyadh, Kingdom of Saudi Arabia.

E-mail: kabbanim@ngha.med.sa lesions. Symptoms and signs of a child presenting with a cardiac emergency may mimic other noncardiac diseases. This may lead to late diagnosis or delay in initiating early management.

Why a child with congenital heart lesion may present in urgent critical condition? And how does he or she present? What are the cardiac diseases that may present as emergencies? How can we make a diagnosis and what are the important steps in management? To answer all these questions, it is important to understand first what are the changes that happen to cardiovascular system during the transition from fetal to neonatal life.

\section{CARDIOVASCULAR CHANGES DURING TRANSITION FROM FETAL TO NEONATAL LIFE}

The cardiorespiratory system goes through multiple changes during the transition from the fetal life to the neonatal

This is an open access article distributed under the terms of the Creative Commons Attribution-NonCommercial-ShareAlike 3.0 License, which allows others to remix, tweak, and build upon the work non-commercially, as long as the author is credited and the new creations are licensed under the identical terms.

For reprints contact: reprints@ @medknow.com

Cite this article as: Kabbani N, Kabbani MS, Al Taweel H. Cardiac emergencies in neonates and young infants. Avicenna J Med 2017;7:1-6. 
life. During fetal life, the lungs are collapsed, their alveoli are filled with lung liquid (LL), and pulmonary vascular resistance (PVR) is high with minimal pulmonary blood flow (Qp). The alveoli that have liquid/blood interface do not participate in gas exchange, and thus, fetus depends entirely on placenta for oxygenation and ventilation. Furthermore, the oxygenated blood coming from placenta through umbilical vein reaches right atrium, and from there, only small part is pumped into the right ventricle while most of the oxygenated blood flow to left atrium (LA) through patent foramen oval (PFO) in the presence of the eustachian valve that directs the oxygenated blood across the PFO and into the LA. ${ }^{[3]}$ The high PVR drives the blood from pulmonary circulation to systemic circulation through patent ductus arteriosus (PDA). During labor, there is a surge of hormones such as epinephrine, vasopressin, cortisol, and thyroid hormones that contribute to the cessation of LL production during labor. ${ }^{[4]}$ With the first gasp of air at birth, lungs expand, remaining LL get absorbed, and air fills alveoli establishing gas/blood interface instead of liquid/blood interface during fetal life. With lung expansion and initiation of effective alveolar gas exchange, Qp increases and PVR decreases with functional cessation of shunt through PDA and PFO leading to the institution of serial pulmonary and systemic circulation. After birth, the ductus arteriosus closes functionally within $12 \mathrm{~h}$ and closes anatomically in $2-3$ weeks to become ligamentum arteriosum. ${ }^{[5]}$

When a baby has congenital heart disease associated with interruption of serial blood flow such as transposition of great arteries (TGAs), pulmonary atresia, mitral atresia, severe coarctation, or interruption of the aorta, the newborn depends on the presence of intercommunication such as PDA and PFO between systemic and pulmonary circulation for survival. Subsequently, when PDA starts to close after birth as it supposes to happen, the baby may collapse due to low cardiac output or severe desaturation. Interventions to maintain PDA open or increase the size of restricted PFO might be lifesaving intervention to keep newborn with ductal-dependent heart lesion alive.
There are many classifications of congenital heart diseases. In this review, we elected to present the congenital heart diseases based on their presentation as a critical acute condition that may be faced by pediatrician, emergency room physician, pediatric cardiologist, or pediatric intensivist. For this purpose, we divided the presentation into four main categories:

- Critical acyanotic heart diseases

- Critical cyanotic heart diseases

- Dysrhythmia presenting as emergency in neonatal stage

- Critical acquired heart diseases presenting in early infancy.

The main focus of this article is to review critical congenital heart diseases as they are the main causes of cardiac emergencies during the neonatal stage and early infancy.

\section{Critical acyanotic heart diseases}

An important category in congenital heart diseases is acyanotic heart diseases. Patients with acyanotic heart diseases may present in critical condition either because of left-sided obstructive lesion with ductal-dependent flow or due to heart failure.

Critical acyanotic ductal-dependent heart diseases In this category of diseases, the normal flow from the left ventricle to systemic arterial circulation may be compromised because of either stenosis or complete interruption of flow. The PDA maintains flow to systemic circulation bypassing the area of stenosis or interruption. When PDA closes, the flow to descending aorta that depends on PDA will be comprised leading to tissue hypoperfusion and tissue hypoxia with subsequent lactic acidosis and shock. ${ }^{[3]}$ A common example of this presentation is neonatal coarctation of the aorta. Newborn with significant coarctation depends on PDA to provide blood flow to descending aorta distal to coarctation [Figure 1]. When PDA starts to close, the infant may present in shock due to a significant decrease in blood flow to lower part of the body in addition to heart failure as the result of increased left

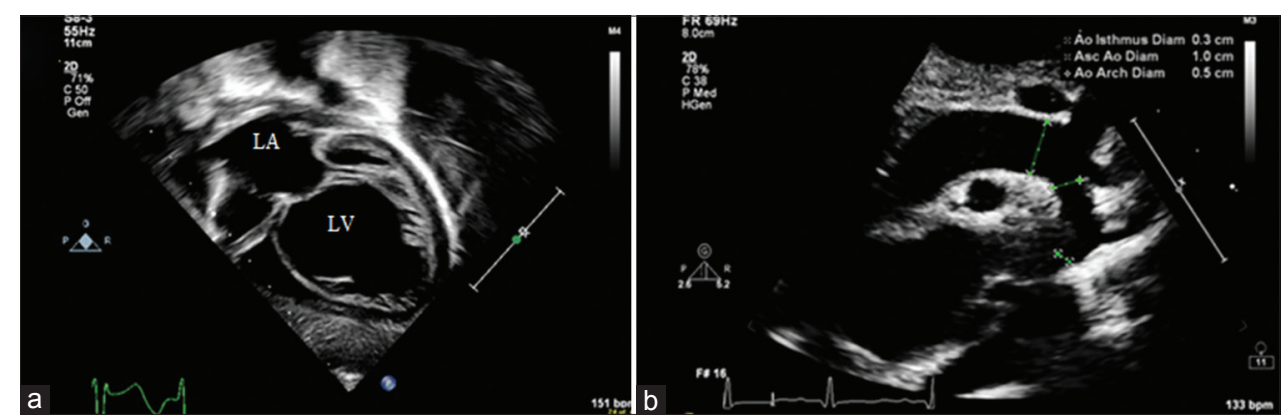

Figure 1: (a) Echocardiography apical view showing dilated left ventricle due to aortic coarctation. (b) Echocardiography showing aortic coarctation in suprasternal view. LA: Left atrium, LV: Left ventricle, Ao: Aorta, Asc: Ascending 
ventricle afterload. It is not unusual for these patients with a left-sided obstructive lesion to present in heart failure with significantly depressed cardiac function, low shortening fraction, increased left ventricle end-diastolic volume, and severe pulmonary edema. Without proper medical or surgical intervention, an infant with severe coarctation may deteriorate rapidly and death may occur. ${ }^{[6,7]}$ When any newborn presents in shock during the first month of life, septic and hypovolemic shock should be excluded as they are more common causes of shock in general, then the four most likely causes of cardiogenic shock have to be ruled out that include: Congenital heart disease with left heart obstruction, cardiac muscle disorders, dysrhythmias, and cardiac metabolic disorders. ${ }^{[8]}$ Coarctation of aorta, and less commonly other left-sided obstructive cardiac lesions such as interrupted aortic arch, critical aortic stenosis, and aortic valve atresia, is the most common cause of congestive heart failure which may progress to shock during the first month of life. Management of neonate with suspected ductal-dependent acyanotic heart lesion should include hemodynamic stabilization, securing airway, connecting to mechanical ventilator to reduce oxygen consumption, intravenous access, and initiation of prostaglandin E1 (PGE1) infusion as soon as possible without delay for echocardiography (ECHO) confirmation or cardiologist consultation as it is lifesaving drug needed to open ductus arteriosus, improve the systemic blood flow and perfusion. As soon as the diagnosis is confirmed and the infant gets stabilized, arrangements should be made aiming to correct the cardiac lesion surgically or by catheter intervention as indicated.

\section{Critical acyanotic heart diseases with heart failure}

In this category of diseases, the child suffers from left to right shunt with increased pulmonary blood flow leading to heart failure. In general, PVR drops after birth as a result of increasing $\mathrm{PaO}_{2}$ due to lungs expansion and starting ventilation with air. ${ }^{[9]}$ In the presence of congenital heart disease that permits left to right shunt such as ventricle septal defect (VSD), atrioventricular septal defect (AVSD), or PDA, the degree of the shunt is directly proportional to the pressure gradient between the two chambers or vessels where the shunt occurs. During the neonatal stage, the PVR is relatively high that will restrict the shunt from left side (or systemic circulation) to the right side (or pulmonary circulation). As the baby grows beyond the first 4-6 weeks of life, PVR gradually drops and gradient between systemic and pulmonary circulation increases leading to more shunt and further pulmonary bloods flow. As a result, the lungs become gradually congested with increasing tachypnea and increased risk of respiratory infection [Figure 2]. A lot of nutritional energy needed for baby growth is consumed

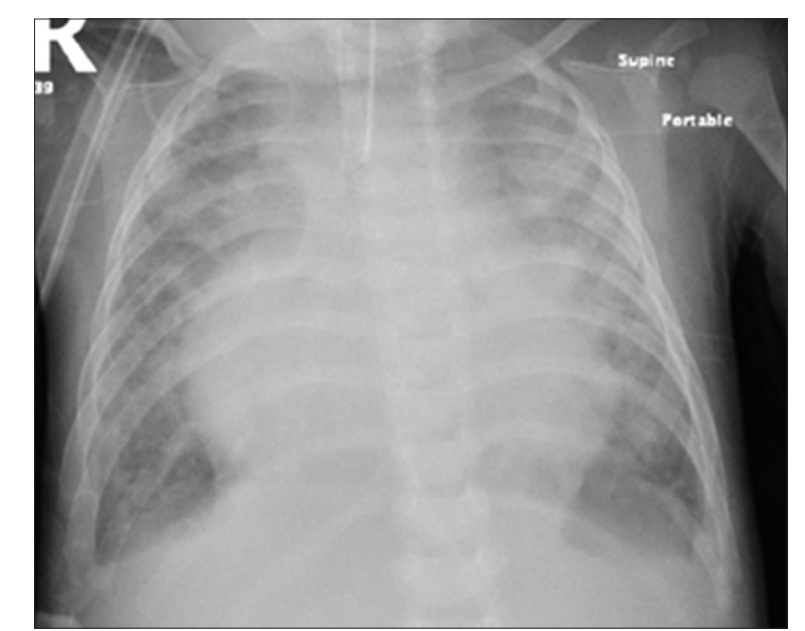

Figure 2: Chest X-ray showing cardiomegaly, significant lungs congestion in infant with atrioventricular septal defect and respiratory syncytial virus infection

by respiratory muscles struggling with increased work of breathing and by heart muscle trying to meet the body $\mathrm{O}_{2}$ demand. Furthermore, the baby with increased work of breathing and heart failure becomes easily tired from any extra effort, so feeding and sucking efforts are frequently interrupted and become associated with sweating and poor oral intake. The combination of previous factors leads to failure to thrive and poor weight gain. An infant with VSD, AVSD, or PDA may present in an emergency or critical condition with decompensated heart failure or respiratory failure due to respiratory infection associated with heart failure. The degree of shunt is related to the pressure gradient between the left ventricle and right ventricle in case of VSD. This shunt is minimal in cases of atrial septal defect (ASD) due to the minimal gradient between the left and right atria that explains why symptoms of heart failure and increased blood flow from ASD do not appear generally in infancy or early childhood. Management of these cases requires immediate stabilization, use of positive pressure ventilation as indicated, diuretics, and reducing afterload using angiotensin-converting enzyme inhibitor or phosphodiesterase III inhibitor. In cases of severe heart failure, inotropes might be needed. Cardiac surgery to eliminate the shunt remains the ultimate treatment once the child is stabilized and acute infection is treated.

\section{Critical cyanotic heart disease}

Cyanotic heart disease is another entity of congenital heart diseases. The cyanosis results from the presence of deoxygenated hemoglobin more than $5 \mathrm{~g} / \mathrm{dL}$. The ability to recognize cyanosis is related to the level of hemoglobin. With higher hemoglobin concentration, we can recognize cyanosis easier. ${ }^{[10]}$ Cyanosis can be peripheral or central. The central cyanosis involves all body and it is recognizable in the mucosa and conjunctiva and requires a thoroughly 
clinical assessment to identify its causes. When a baby presents with cyanosis or desaturation, the physician should look for causes that lead to the presence of deoxygenated hemoglobin in the blood. In general, this may be due to one of the three causes: (1) Incomplete oxygenation process of blood passing through the lung due to lung pathology leading to intrapulmonary shunt, (2) or due to shunting of deoxygenated blood from the right side of the heart to the left side systemic circulation leading to intracardiac shunt, or (3) due to having abnormal hemoglobin that cannot bind to oxygen. Examples of the first cause where the blood does not get fully oxygenated in the lungs as it happens in cases of collapsed lung or in cases of alveolar diseases such as pulmonary edema, respiratory distress syndrome, or severe pneumonia. This type of ventilation/perfusion (V/Q) mismatching is the result of intrapulmonary shunt. The saturation of patient with intrapulmonary shunt may improve with supplementing oxygen and positive pressure ventilation as it improves V/Q matching. The second type of shunt that can lead to the presence of deoxygenated blood in systemic circulation is intracardiac shunt that is present when the blood coming from venous circulation (right side of heart) crosses to systemic circulation (left side of heart) through abnormal communication within heart chamber or great vessels. An example of this intracardiac shunt is Tetralogy of Fallot (TOF) where the venous blood coming to the right side of the heart crosses to the left side through VSD in association with right ventricle outflow obstruction. When deoxygenated blood is mixed with oxygenated blood, the resulted blood has a lower saturation than normal and may lead to significant central cyanosis. This type of shunt is called intracardiac shunt. One of clinical bedside tests that can help differentiating intrapulmonary from intracardiac shunt is by giving $100 \% \mathrm{FiO}_{2}$ through oxygen hood to the affected neonate. The peripheral saturation usually improves in cases of lung diseases associated with intrapulmonary shunt; while on the other hand, the cyanosis, $\mathrm{O}_{2}$ saturation, and $\mathrm{PaO}_{2}$ do not improve in cases of intracardiac shunt due to congenital heart diseases. The shunt may occur through PDA in cases of persistent pulmonary hypertension of neonates (PPHN) leading to shunt from pulmonary artery to aorta through PDA. In this case, the shunt and saturation may partially improve with supplementing $100 \% \mathrm{FiO}_{2}$ that has a vasodilatory effect on the pulmonary vascular bed. Measuring preductal saturation (right arm) and postductal saturation (left leg) may show a significant difference between pre- and post-ductal $\mathrm{PaO}_{2}$ indicating that the blood is shunted at the level of PDA from pulmonary to systemic circulation as it happens in cases of PPHN.

Why and when a neonate with cyanotic heart disease may present in critical condition? There are five common cyanotic congenital heart diseases known as (5Ts) that may present in an emergency or critical condition.

1. The first of these diseases is TGAs. In TGA, the aorta originates from right ventricle while pulmonary artery originates from left ventricle. This condition is not compatible with life unless there is intercommunication between systemic and pulmonary circulation. Baby born with TGA may present with severe cyanosis and hemodynamic instability, particularly when the PDA starts to close. PGE1 is a lifesaving medication that needs to be started to maintain PDA. However, the oxygenated blood will return to LA and from there should be shunted through PFO to the right atrium to provide the systemic circulation with oxygenated blood. If foramen oval is small and restrictive, the baby may present with severe cyanosis, acidosis, lung congestion, and hemodynamic instability. ECHO can help defining the size of foramen oval, the presence of any gradient though PFO, or the presence of atrial septum right shifting indicating the tension of blood in LA and the need for urgent lifesaving atrial septostomy. This ECHO finding is often associated with hemodynamic instabilities and signs of inadequate cardiac output such as lactic acidosis, poor perfusion, hypotension, decreased urine output, and low level of $\mathrm{PaO}_{2}$ in arterial blood. Once the baby is stabilized with balloon atrial septostomy, the baby can be referred to surgery that should be done within the first 3 weeks of life before the left ventricle deconditioning takes place due to a decrease in PVR

2. The second disease that may present with cyanosis is TOF. In TOF, ECHO shows right ventricular outflow obstruction due to infundibular hypertrophy, overriding aorta, VSD, and right ventricle hypertrophy, and chest X-ray (CXR) may show boot-shaped cardiac silhouette [Figure 3]. Neonates with TOF usually do

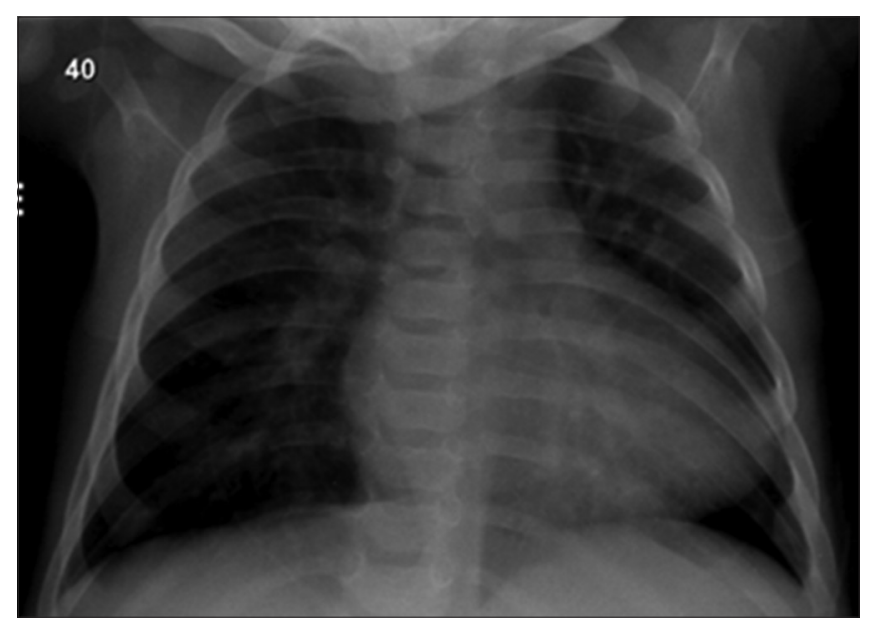

Figure 3: Chest X-ray showing boot-shaped cardiac silhouette with oligemic lung in infant with Tetralogy of Fallot 
not present as an emergency during the first month of life unless the pulmonary blood flow is significantly compromised as a result of severe right ventricle outflow tract (RVOT) obstruction. This in principal is similar to pulmonary atresia where the RVOT is completely occluded, and blood flow to the lung should be maintained by PDA. PGE1 should be started to maintain PDA until surgery or intervention is performed with the aim to augment pulmonary blood flow. An infant with TOF, however, may present during infancy with a critical condition known as hypercyanotic spell (Tet spell). In Tet spell, the right ventricle outflow spasms that result in significant decrease in pulmonary blood flow and increase shunt of deoxygenated blood to systemic circulation through VSD. The infant may become deeply blue, limb with severe acidosis due to tissue hypoxia that may threaten the life. Measures that should be taken immediately are airway support with oxygen, hydration, correction of acidosis, and minimize right to left shunt by increasing systemic vascular resistance. Phenylephrine can be used to increase SVR and morphine sulfate can also help to relax RVOT. Once the child is stabilized, corrective surgery should be performed to release RVOT obstruction and close VSD

3. The third disease in this category is tricuspid atresia (TA). Some of these patients may have an intact ventricular septum and they depend completely on PDA to maintain pulmonary blood flow. There are a group of patients who may have an associated VSD that help in providing forward pulmonary blood flow. The lungs in the latter group are usually congested due to increased pulmonary blood flow. Patient with TA-intact ventricular septum (TA-intact septum) may present as an emergency in neonatal stage due to decrease pulmonary blood flow and cyanosis that require immediate opening of PDA using PGE1. On the other hand, patients with TA-VSD may present due lung congestion associated with cyanosis and increase work of breathing that requires restriction of pulmonary blood flow

4. The fourth disease in the category of cyanotic heart disease that may present as emergency is truncus arteriosus. Both aorta and pulmonary artery originate from a single trunk. The lungs are congested due to increased pulmonary blood flow and infant presents in heart failure with respiratory distress. These patients require corrective surgery that establishes a connection between the left ventricle and neo-aorta and between the right ventricle and pulmonary artery through conduit placement (Rastelli operation) with the closure of VSD

5. The fifth disease in this category is total anomalous pulmonary venous drainage. In this disease, pulmonary veins are connected through a vertical vein to superior vena cava (SVC) or to coronary sinus or to inferior vena cava (IVC). The junction of abnormally connected vein to IVC, SVC, or coronary sinus might be narrow leading to obstruction of pulmonary venous drainage. This will be associated with cyanosis, severe lung congestion, and decreased cardiac output. Neonate may present with severe respiratory failure, acidosis, and hemodynamic instability. Those patients are true surgical cardiac emergency and require immediate surgical correction to establish a proper connection between pulmonary veins and LA with no obstruction.

\section{Dysrhythmia presenting as emergency in neonatal stage}

Neonates may present with tachy or bradyarrhythmia. Dysrhythmia can be associated with normal structure heart or abnormal heart. Neonates of mother with lupus erythematous are known to be at risk for congenital complete heart block [Figure 4]. Furthermore, infant with corrected TGAs is also known to be at risk for complete heart block. Management of these cases depends on the presentation and hemodynamic instability.

\section{Acquired congenital heart diseases}

Young infants may present with the critical cardiac condition due to acquired diseases. Myocarditis and cardiomyopathy may affect young infant leading to depressed function and cardiogenic shock. Kawasaki disease is another acquired disease that may affect the heart leading to coronary aneurysm formation. Infectious causes leading to endocarditis, pericarditis, or pericardial effusion are also possible unusual causes for pediatric cardiac emergency presentation in early infancy.

\section{CONCLUSION}

Neonates and young infants may present with cardiac emergencies related mainly to congenital heart diseases.

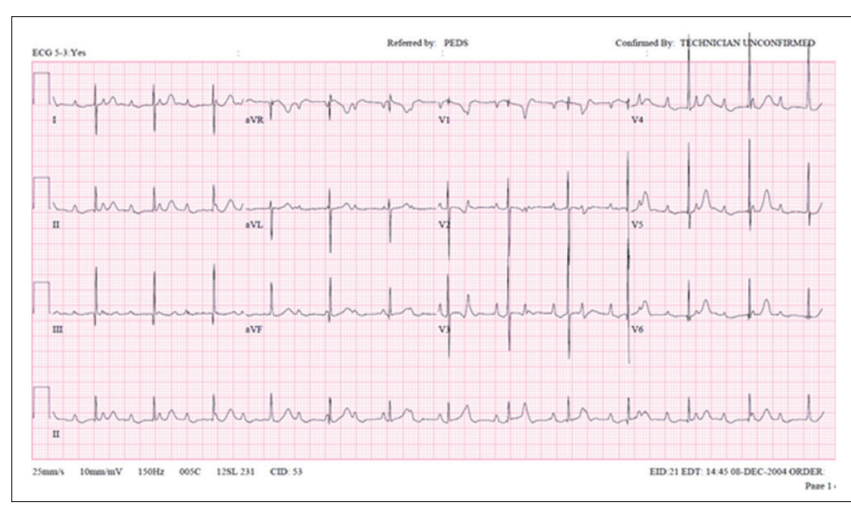

Figure 4: Electrocardiogram shows complete heart block in a 5-day-old neonate of mother with lupus erythematosus 
Ductus-dependent lesions are the main cause for these types of emergencies. Early recognition and treatment are essential for lifesaving and for reserving cardiac function to achieve the best outcome after repair.

\section{Financial support and sponsorship}

Nil.

\section{Conflicts of interest}

There are no conflicts of interest.

\section{REFERENCES}

1. Tsao PC, Shiau YS, Chiang SH, Ho HC, Liu YL, Chung YF, et al. Development of a newborn screening program for critical congenital heart disease (CCHD) in Taipei. PLoS One 2016;11:e0153407.

2. Meyer S, Grundmann U, Reinert J, Gortner L. Specific aspects of pediatric emergencies in the prehospital setting. Med Klin Intensivmed Notfmed 2016;111:65-77.

3. Watson T, Kakar P, Srivastava S, Dhanjal TS. Eustachian valve remnant. Cardiol J 2007;14:508-9.

4. Kabbani MS, Cassin S. The effects of cGMP on fetal sheep pulmonary blood flow and lung liquid production. Pediatr Res 1998;43:325-30.

5. Anilkumar M. Patent ductus arteriosus. Cardiol Clin 2013;31:417-30.

6. Rosenthal E. Coarctation of the aorta from fetus to adult: Curable condition or life long disease process? Heart 2005;91:1495-502.

7. Ward KE, Pryor RW, Matson JR, Razook JD, Thompson WM, Elkins RC. Delayed detection of coarctation in infancy: Implications for timing of newborn follow-up. Pediatrics 1990;86:972-6.

8. Albrecht GT. Cardiogenic shock in the neonate. Indian J Pediatr 1993;60:381-91.

9. Rudolph AM. High pulmonary vascular resistance after birth: I. Pathophysiologic considerations and etiologic classification. Clin Pediatr (Phila) 1980;19:585-90.

10. Martin L, Khalil $\mathrm{H}$. How much reduced hemoglobin is necessary to generate central cyanosis? Chest 1990;97:182-5. 